\title{
New Fusarium Head Blight-Resistant Sources from Asian Wheat Germplasm
}

\author{
Jian-Bin Yu, Gui-Hua Bai,` Shi-Bin Cai, Yan-Hong Dong, and Tomohiro Ban
}

\begin{abstract}
Fusarium head blight (FHB) is an important disease of wheat worldwide. Severe infection can dramatically reduce grain yield and quality. Resistant cultivars have been identified from several countries. However, only a few sources of $\mathrm{FHB}$ resistance showed stable $\mathrm{FHB}$ resistance across environments and have been used as the major source of resistance in breeding programs. To diversify the wheat FHB-resistance gene pool, new sources of FHB-resistance are desired. Ninety-four selected wheat landraces and cultivars, mainly from China and Japan, have been evaluated for FHB severity and deoxynivalenol (DON) content. Low DON content was correlated with resistance to the FHB symptom spread within an infected spike, but not with the resistance to FHB initial infection. Two-thirds of the accessions were either resistant or moderately resistant to FHB. Among them, 26 highly resistant accessions mainly originated from China and Japan. Fifteen of them had less than $2 \mathrm{mg} \mathrm{kg}^{-1} \mathrm{DON}$ in harvested grain, six of which showed all three types of resistance. Most of these resistant accessions lack known pedigree relations to Sumai 3 , suggesting that some of them may carry genes for resistance to FHB and DON accumulation different from those in Sumai 3.
\end{abstract}

Jian-Bin Yu, Department of Agronomy, and Gui-Hua Bai, USDAARS-PSERU, Kansas State University, Manhattan, KS, 66506; ShiBin Cai, Jiangsu Academy of Agricultural Sciences, Nanjing, 210014, China; Yan-Hong Dong, Department of Plant Pathology, University of Minnesota, St. Paul, MN, 55108; Tomohiro Ban, Kihara Institute for Biological Research, Yokohama City University, Yokohama 244-0813, Japan. Research is partly funded by the U.S. Wheat and Barley Scab Initiative and the National Research Initiative of the USDA Cooperative State Research, Education and Extension Service, Coordinated Agricultural Project grant number 2006-55606-16629. Mention of trade names or commercial products in this article is solely for the purpose of providing specific information and does not imply recommendation or endorsement by the U.S. Department of Agriculture. This is contribution No. 08-170-J from the Kansas Agricultural Experiment Station, Manhattan, Kansas. Received 27 Dec. 2007. ${ }^{\star}$ Corresponding author (guihua.bai@ars.usda.gov).

Abbreviations: d.a.i., days after inoculation; DON, deoxynivalenol; FHB, Fusarium head blight; PIF, percentage of infected florets; PSS, percentage of symptomatic spikelets.

$\mathrm{F}$ USARIUm head blight (FHB), or scab, is one of the most severe diseases in cereal crops grown in humid and semihumid areas worldwide (Parry et al., 1995). It is mainly caused by F. graminearum Schwabe in the U.S. and many other countries (Bai and Shaner, 2004). When warm and wet weather coincides with wheat anthesis and abundant natural inocula, severe FHB epidemics may occur and can dramatically reduce grain yield and quality (Bai and Shaner, 1994; McMullen et al., 1997). In addition, infected grain is often contaminated with deoxynivalenol (DON), a mycotoxin produced by F. graminearum. DON contamination in

Published in Crop Sci. 48:1090-1097 (2008).

doi: 10.2135/cropsci2007.10.0554

(C) Crop Science Society of America

677 S. Segoe Rd., Madison, WI 53711 USA

All rights reserved. No part of this periodical may be reproduced or transmitted in any form or by any means, electronic or mechanical, including photocopying, recording, or any information storage and retrieval system, without permission in writing from the publisher. Permission for printing and for reprinting the material contained herein has been obtained by the publisher. 
kernels of wheat and other small grain crops has become a major concern for animal production and human health (Bai et al., 2001b; Luo et al., 1990; Marasas et al., 1984). The maximum DON levels in wheat grain allowable for human consumption range from 0.5 to $2 \mathrm{mg} \mathrm{kg}^{-1}$ in the U.S., Canada, and some European countries (Dexter and Nowicki, 2003; Snijders, 1990).

The use of resistant cultivars together with appropriate crop management practices is the most commonly employed strategy for the control of FHB disease (Bai and Shaner, 1996). Resistance to FHB in wheat is polygenic. Three major types of host resistance have been reported, host resistance to the initial infection by the fungus (type I), resistance to the spread of FHB symptoms within an infected spike (type II), and resistance to accumulation of mycotoxin in infected grains (type III) (Mesterházy, 1995; Miller et al., 1985; Schroeder and Christensen, 1963). Type II resistance is the predominant type of resistance identified in the majority of wheat cultivars.

FHB-resistant cultivars have been identified from different geographic regions, including the U.S., Asia, Europe, and South America (Bai and Shaner, 2004). However, only a few wheat lines, such as Sumai 3 and its derivatives, have been shown to contain type II resistance that is effective in a range of environments and extensively used as a major FHB-resistant source in breeding programs worldwide (Bai and Shaner, 2004; Rudd et al., 2001). New and diverse sources of FHB-resistance genes may facilitate the achievement of high levels of potentially durable FHB resistance in wheat cultivars.

Low DON content has long been proposed as type III resistance (Miller et al., 1985). But the relationship between the FHB disease severity and DON content in harvested grain is still not conclusive (Bai et al., 2001b; Shaner and Buechley, 2003). Some studies reported a low correlation between FHB severity and DON content (Miedaner et al., 2003), whereas others reported that FHB-resistant wheat cultivars often had low DON content in harvested grain. Wheat cultivars with very low or no DON are very desirable for growers to reduce economic losses caused by FHB epidemics (Bai and Shaner, 2004). In this study, wheat genotypes with different levels of FHB resistance from diverse origins were evaluated under favorable FHB epidemic conditions. The objectives of this study were to (i) characterize different types of resistance in Asian landraces and cultivars, (ii) identify new sources of FHB resistance by evaluating the genotypes for all three types of FHB resistance, and (iii) investigate the relationship between FHB disease severity and DON content.

\section{MATERIALS AND METHODS}

\section{Plant Materials and Pathogen Inoculum}

Ninety-four landraces, cultivars, or breeding lines were evaluated for three types of resistance in repeated greenhouse experiments (Table 1). Among these accessions, 63 Chinese accessions were acquired from the USDA-ARS National Small Grains Collection at Aberdeen, ID, and the Jiangsu Academy of Agricultural Sciences, Nanjing, China; 24 Japanese accessions were provided by the Gene Bank of Japan; one Korean cultivar was provided by Dr. G. E. Shaner at Purdue University, IN. Five cultivars from the U.S. and other countries were used as references for various levels of FHB resistance. Sumai 3 and Wheaton were used as resistant and susceptible controls, respectively (Table 1). The inoculum was a field isolate of $F$. graminearum (GZ 3639) that originated from Kansas (Desjardins et al., 1996) and was verified for stability of virulence pattern and DON accumulation by annual inoculation of a set of wheat differentials.

\section{FHB Resistance Evaluation}

FHB resistance of all the wheat accessions was evaluated in the greenhouse at Kansas State University from 2003 to 2005. FHB conidial inoculum was prepared with mung bean liquid medium (Bai and Shaner, 1996) and used to inoculate wheat plants at anthesis (Feekes Growth Stage 10.51). Type II resistance was evaluated by injecting $10 \mu \mathrm{L}$ of conidiospores of F. graminearum $(\sim 100$ spores/ul) into a central floret of a spike using a syringe; type I resistance was evaluated by spraying $2 \mathrm{~mL}$ of conidiospore suspension ( 1000 conidiospores $/ \mathrm{ml})$ on each spike. The plants were prepared for inoculation as follows: after vernalization at $4^{\circ} \mathrm{C}$ in a growth chamber for $8 \mathrm{wk}$, six seedlings were transplanted into a 5 " $\times 5$ " Dura-pot (Hummert Int., Earth City, MO) containing Metro-mix 360 soil mix (Hummert Int., Earth City, MO), and grown in a greenhouse with a $12 \mathrm{~h}$ daylight period. One spike per plant was inoculated, and inoculated plants were enclosed in a moist chamber at $25 \pm 5^{\circ} \mathrm{C}$ with a 12-h daylight period for $3 \mathrm{~d}$ to promote initial infection. The pots were moved to random positions on the greenhouse bench following a complete randomized design, and grown at $25 \pm$ $5^{\circ} \mathrm{C}$ during day and $17 \pm 2{ }^{\circ} \mathrm{C}$ during night. Type I resistance was evaluated as the percentage of infected florets (PIF) in an inoculated spike $7 \mathrm{~d}$ after inoculation (d.a.i) to avoid the spread of infection from initially infected florets to neighbor florets (type II resistance). For type II resistance, the infected and total numbers of spikelets in an inoculated spike were counted on the 21st d.a.i., and the percentage of symptomatic spikelets (PSS) was calculated as final disease severity. Mean values of the disease ratings based on entries were used for data analysis. Type I resistance was evaluated in 2004 and 2005 experiments and type II resistance was evaluated in the spring and fall experiments of 2003 with three replicates in each of the experiments. Each replicate consists of one pot of transplanted plants.

\section{DON Determination}

For each accession, inoculated spikes were carefully harvested and threshed manually to save all grains including highly infected, shriveled ones. Grains from the three replicates of each genotype in each experiment were combined for toxin analysis. DON content was determined as the amount of DON (mg $\mathrm{kg}^{-1}$ ) in the wheat kernels harvested from Fusarium-inoculated spikes by gas chromatography-mass spectrometry (GC-MS) according to Mirocha et al. (1998). 
Table 1. Entry mean values of percentage of infected florets (PIF) in a spike, percentage of symptomatic spikelets (PSS) in a spike, and deoxynivalenol content (DON) in $\mathrm{mg} \mathrm{kg}^{-1}$ for 94 wheat accessions from different origins based on greenhouse-grown plants.

\begin{tabular}{|c|c|c|c|c|c|c|c|c|}
\hline \multirow{2}{*}{ Accession name } & \multirow{2}{*}{ Country } & \multirow{2}{*}{ Source $^{\dagger}$} & \multicolumn{2}{|c|}{ Spraying-inoculation } & \multicolumn{2}{|c|}{ Single point-inoculation } & \multirow{2}{*}{$\begin{array}{l}\text { Combined } \\
\text { DON }\end{array}$} & \multirow{2}{*}{ Rating? } \\
\hline & & & PIF & DON & PSS & DON & & \\
\hline & & & $\%$ & $\mathrm{mg} \mathrm{kg}^{-1}$ & $\%$ & $\mathrm{mg} \mathrm{kg}^{-1}$ & $\mathrm{mg} \mathrm{kg}^{-1}$ & \\
\hline Ning 7840 & China & JAAS & 30.1 & 0.8 & 6.6 & 0.6 & 0.7 & $\mathrm{R}$ \\
\hline Huoshaobairimai & China & JAAS & 17.8 & 1.0 & 22.0 & 1.6 & 1.3 & $\mathrm{R}$ \\
\hline Asozaira III & Japan & JIRCAS & 23.6 & 1.1 & 8.9 & 2.0 & 1.5 & $\mathrm{R}$ \\
\hline Nobeokabozu & Japan & PI382153 & 24.5 & 1.6 & 24.3 & 1.5 & 1.5 & $\mathrm{R}$ \\
\hline Asozairai & Japan & JIRCAS & 32.1 & 1.7 & 12.7 & 1.6 & 1.6 & $\mathrm{R}$ \\
\hline Huangfangzhu & China & JAAS & 24.1 & 1.4 & 20.2 & 2.1 & 1.6 & $\mathrm{R}$ \\
\hline Huangcandou & China & JAAS & 12.2 & 2.3 & 13.4 & 1.1 & 1.7 & $\mathrm{R}$ \\
\hline Huoshaomai & China & JAAS & 17.3 & 0.7 & 21.4 & 2.6 & 1.7 & $\mathrm{R}$ \\
\hline Fu 5114 & China & JAAS & 33.2 & 4.7 & 7.4 & 0.4 & 1.8 & $\mathrm{R}$ \\
\hline Qiaomai Xiaomai & Japan & JIRCAS & 40.0 & 3.1 & 22.4 & 1.0 & 2.1 & $\mathrm{R}$ \\
\hline Baisanyuehuang & China & JAAS & 23.3 & 3.3 & 12.5 & 1.3 & 2.3 & $\mathrm{R}$ \\
\hline F 60096 & China & JAAS & 20.9 & 2.1 & 6.9 & 3.2 & 2.6 & $\mathrm{R}$ \\
\hline 'Yangmai 1' & China & JAAS & 23.6 & 3.5 & 22.5 & 1.8 & 2.6 & $\mathrm{R}$ \\
\hline Minamikyushu 69 & Japan & PI382152 & 31.7 & 3.2 & 7.4 & 2.4 & 2.9 & $\mathrm{R}$ \\
\hline Wangshuibai & China & JAAS & 15.7 & 9.5 & 8.7 & 0.7 & 3.6 & $\mathrm{R}$ \\
\hline Mutanchiang & China & PI70675 & 25.1 & 3.7 & 21.4 & - & 3.7 & $\mathrm{R}$ \\
\hline Haiyanzhong & China & JAAS & 20.9 & 4.4 & 13.7 & 3.2 & 3.8 & $\mathrm{R}$ \\
\hline Fumai 3 & China & JAAS & 21.3 & 3.0 & 18.6 & 5.5 & 4.2 & $\mathrm{R}$ \\
\hline Linghaimaoyangmo & China & $\mathrm{Pl} 435124$ & 23.1 & 1.7 & 9.3 & 7.0 & 4.3 & $\mathrm{R}$ \\
\hline Taiwan Wheat & China & JAAS & 23.8 & 11.0 & 13.0 & 0.9 & 6.0 & $\mathrm{R}$ \\
\hline Caizihuang & China & JAAS & 28.8 & 4.1 & 22.0 & 9.2 & 6.6 & $\mathrm{R}$ \\
\hline Chukoku 81 & Korea & Indiana & 27.0 & 8.5 & 19.5 & 8.2 & 8.4 & $\mathrm{R}$ \\
\hline Su 49 & China & JAAS & 20.3 & 20.3 & 7.8 & 2.7 & 8.6 & $\mathrm{R}$ \\
\hline Shirasaya No1 & Japan & Pl197129 & 19.8 & 13.0 & 22.4 & 4.2 & 8.6 & $\mathrm{R}$ \\
\hline ‘Ernie’ & USA & PI592001 & 24.9 & 16.5 & 20.5 & 2.3 & 9.4 & $\mathrm{R}$ \\
\hline Meiqianwu & China & PI525071 & 25.3 & $-^{\ddagger}$ & 16.1 & 9.5 & 9.5 & $\mathrm{R}$ \\
\hline Tokai 66 & Japan & PI382161 & 25.7 & 0.8 & 35.4 & 2.6 & 1.0 & MR \\
\hline Nyubai & Japan & JIRCAS & 32.0 & 0.8 & 40.2 & 1.6 & 1.1 & MR \\
\hline Shoukomugi II & Japan & JIRCAS & 37.9 & 1.5 & 26.3 & 1.7 & 1.6 & MR \\
\hline 'Yangmai 158' & China & JAAS & 28.6 & 2.8 & 30.3 & 0.7 & 1.8 & MR \\
\hline Itoukomugi & Japan & JIRCAS & 24.1 & 2.9 & 30.1 & 0.9 & 1.9 & MR \\
\hline Sotome & Japan & JIRCAS & 24.8 & 1.5 & 42.7 & 3.8 & 2.2 & MR \\
\hline Sobakomugi 1B & Japan & JIRCAS & 28.0 & 1.6 & 32.4 & 3.8 & 2.7 & MR \\
\hline Chokwang & Korea & JAAS & 24.0 & 3.0 & 28.7 & 3.1 & 3.1 & MR \\
\hline Sotome A & Japan & JIRCAS & 43.5 & 1.5 & 32.5 & 3.8 & 3.1 & MR \\
\hline Nobeokabozu Komugi & Japan & JIRCAS & 40.0 & 4.0 & 32.3 & 2.0 & 3.3 & MR \\
\hline Yanglazi & China & PI502932 & 31.2 & 4.1 & 39.1 & 6.0 & 3.5 & MR \\
\hline 'Wannin 2' & China & JAAS & 22.7 & 3.3 & 44.1 & 3.7 & 3.5 & MR \\
\hline Hongjianzi & China & JAAS & 17.4 & 4.4 & 28.6 & 3.0 & 3.7 & MR \\
\hline 'Freedom' & USA & Pl592002 & 35.2 & 5.6 & 27.8 & 2.0 & 3.8 & $\mathrm{MR}$ \\
\hline Kagoshima & Japan & JIRCAS & 19.5 & 8.6 & 31.2 & 1.0 & 4.8 & MR \\
\hline Shuilizhan & China & PI502930 & 20.0 & 4.3 & 37.3 & 5.7 & 5.0 & MR \\
\hline Qiangshuihuang & China & PI502931 & 26.5 & 6.3 & 28.1 & 8.0 & 5.1 & MR \\
\hline FSW & China & JAAS & 24.5 & 9.5 & 32.2 & 1.4 & 5.4 & MR \\
\hline Huishanyangmai & China & PI462154 & 22.8 & 8.8 & 37.1 & 4.7 & 5.6 & MR \\
\hline Abura & Japan & PI382140 & 23.5 & 2.9 & 36.4 & 8.5 & 5.7 & MR \\
\hline Shinchunaga & Japan & Pl197128 & 30.3 & 10.0 & 39.6 & 1.7 & 5.8 & MR \\
\hline Sobakomugi $1 \mathrm{C}$ & Japan & JIRCAS & 40.4 & 2.2 & 27.1 & 10.5 & 6.3 & MR \\
\hline WZHHS & China & JAAS & 16.9 & 10.0 & 26.9 & 3.1 & 6.6 & MR \\
\hline 'Yangmai 4' & China & JAAS & 26.7 & 9.4 & 34.6 & 5.8 & 7.6 & MR \\
\hline
\end{tabular}


Table 1. Continued.

\begin{tabular}{|c|c|c|c|c|c|c|c|c|}
\hline \multirow{2}{*}{ Accession name } & \multirow{2}{*}{ Country } & \multirow{2}{*}{ Source $^{\dagger}$} & \multicolumn{2}{|c|}{ Spraying-inoculation } & \multicolumn{2}{|c|}{ Single point-inoculation } & \multirow{2}{*}{$\begin{array}{l}\text { Combined } \\
\text { DON }\end{array}$} & \multirow{2}{*}{ Rating? } \\
\hline & & & PIF & DON & PSS & DON & & \\
\hline & & & $\%$ & $\mathrm{mg} \mathrm{kg}^{-1}$ & $\%$ & $\mathrm{mg} \mathrm{kg}^{-1}$ & $\mathrm{mg} \mathrm{kg}^{-1}$ & \\
\hline 'Yangmai 5' & China & JAAS & 28.2 & 10.2 & 35.0 & 6.5 & 8.4 & MR \\
\hline ‘Jiangdongmen’ & China & PI462135 & 33.2 & 9.0 & 36.5 & 11.2 & 10.1 & MR \\
\hline Canlaomai & China & JAAS & 18.4 & 15.9 & 39.4 & 9.8 & 12.8 & MR \\
\hline 'Emai6' & China & JAAS & 17.7 & 26.3 & 29.0 & 3.0 & 14.6 & MR \\
\hline Dahongpao & China & JAAS & 31.5 & 13.3 & 43.3 & 20.2 & 16.7 & MR \\
\hline Xinghuabaiyuhua & China & PI462150 & 16.8 & 13.4 & 43.1 & 21.1 & 17.2 & MR \\
\hline Youbaomai & China & PI524980 & 32.8 & 10.2 & 41.3 & 28.1 & 19.2 & MR \\
\hline Sapporoharukomugijugo & Japan & PI81791 & 21.6 & 2.0 & 52.3 & 2.2 & 2.1 & MS \\
\hline Abura Komugi & Japan & JIRCAS & 28.9 & 2.2 & 48.0 & 3.2 & 2.7 & MS \\
\hline 'Funo' & Italy & JAAS & 29.5 & 1.4 & 51.8 & 5.2 & 3.3 & MS \\
\hline Fangtouhongmang & China & PI502938 & 23.9 & 4.2 & 69.5 & 4.0 & 4.1 & MS \\
\hline Sanshukomugi & Japan & Pl197130 & 12.2 & 4.3 & 49.0 & 5.9 & 5.1 & MS \\
\hline Kikuchi & Japan & JIRCAS & 21.2 & 2.9 & 49.3 & 7.6 & 5.3 & MS \\
\hline Xueliqing & China & JAAS & 21.1 & 4.5 & 47.8 & 7.4 & 6.0 & MS \\
\hline Paimaitze & China & Pl64285 & - & 8.2 & 69.9 & 8.5 & 8.3 & MS \\
\hline Fangtoubaimang & China & PI502935 & - & 4.5 & 68.0 & 12.0 & 9.5 & MS \\
\hline Dafanliuzhu & China & JAAS & 27.5 & 14.1 & 54.4 & 5.2 & 9.6 & MS \\
\hline NTDHP & China & JAAS & 39.7 & 13.2 & 66.6 & 6.6 & 9.9 & MS \\
\hline Sanchaho & China & PI70674 & 37.0 & 4.8 & 56.6 & 15.4 & 10.1 & MS \\
\hline Honghuawu & China & PI502949 & 25.9 & 10.5 & 54.9 & 10.5 & 10.5 & MS \\
\hline Dahuangpi & China & PI502939 & 36.8 & 16.0 & 45.6 & 5.5 & 10.7 & MS \\
\hline Chushanbao & China & PI524973 & 32.2 & 16.1 & 62.5 & 5.2 & 10.7 & MS \\
\hline Liangguangtou & China & PI435109 & 26.0 & 15.9 & 49.0 & 0.9 & 10.9 & MS \\
\hline Chile & Chili & JIRCAS & 30.4 & 9.3 & 57.3 & 12.9 & 11.1 & MS \\
\hline Shanhaicaizihuang & China & PI462140 & 11.2 & 19.2 & 53.5 & 3.3 & 11.2 & MS \\
\hline Heshangmai & China & JAAS & 32.7 & 22.7 & 66.2 & 1.4 & 12.0 & MS \\
\hline 'Jingzhou 1’' & China & JAAS & 23.8 & 16.1 & 69.1 & 11.1 & 13.6 & MS \\
\hline Shironankin & Japan & JIRCAS & 29.0 & 24.4 & 60.5 & 5.8 & 15.1 & MS \\
\hline Hongmongbai & China & PI518598 & - & 48.8 & 64.8 & 0.8 & 24.8 & MS \\
\hline 'Zhen 7495' & China & JAAS & 38.4 & 72.0 & 54.5 & 8.3 & 29.5 & MS \\
\hline Hungguangtou & China & PI447389 & 29.3 & 19.0 & 66.8 & 41.2 & 30.1 & MS \\
\hline Zairai Yuubou & Japan & JIRCAS & 29.2 & 48.8 & 61.8 & 11.9 & 30.4 & MS \\
\hline Fusuihuang & China & JAAS & 32.5 & 77.2 & 49.3 & 6.6 & 41.9 & MS \\
\hline Youzimai & China & PI435110 & 42.3 & 80.0 & 48.1 & 13.3 & 46.6 & MS \\
\hline Kuangtuerhhsiaomai & China & PI57347 & 25.9 & 2.2 & 71.4 & 1.3 & 1.6 & S \\
\hline Hongmangmai & China & PI525072 & - & 7.0 & 76.0 & - & 7.0 & S \\
\hline Nanda 2419 & China & JAAS & 9.1 & 11.1 & 81.5 & 4.4 & 7.7 & $S$ \\
\hline Dabaipao & China & PI525074 & - & 16.9 & 78.5 & 9.6 & 13.2 & S \\
\hline Chinese Spring & China & JAAS & 30.9 & 42.1 & 76.5 & 3.2 & 22.7 & $S$ \\
\hline Sanyuehuang & China & JAAS & 26.1 & 30.6 & 92.2 & 17.9 & 24.2 & S \\
\hline Avrora & Russia & JAAS & 26.5 & 28.3 & 70.2 & 32.5 & 29.7 & $S$ \\
\hline Yulinbai & China & PI591997 & - & 33.8 & 72.3 & 31.2 & 32.5 & $S$ \\
\hline Tafangshen & China & Pl70666 & 25.2 & 12.7 & 82.6 & 62.9 & 37.8 & $S$ \\
\hline Chanjibaidongmai & China & PI445868 & 46.5 & 42.0 & - & 95.9 & 77.9 & S \\
\hline Sumai 3 & China & JAAS & 28.1 & 5.2 & 18.8 & 3.0 & 4.1 & $\mathrm{R}$ \\
\hline Wheaton & USA & PI469271 & 28.2 & 250.6 & 89.2 & 112.7 & 181.6 & S \\
\hline $\mathrm{LSD}_{0.05}$ & & & 16.2 & 76.7 & 22.1 & 40.1 & 30.6 & \\
\hline
\end{tabular}

†JAAS = Jiangsu Academy of Agricultural Science, Nanjing, P.R. China; JIRCAS = Japan International Research Center for Agricultural Sciences.

‡Data missing.

§Mean values based on DON data from both spraying-inoculation and single point-inoculation experiments.

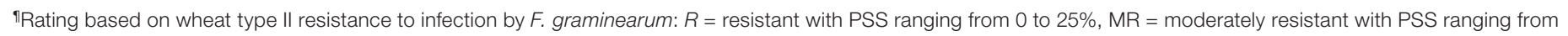
26 to $45 \%, M S=$ moderately susceptible with PSS ranging from 46 to $70 \%$, and S = susceptible with PSS ranging from 71 to $100 \%$. 
a.

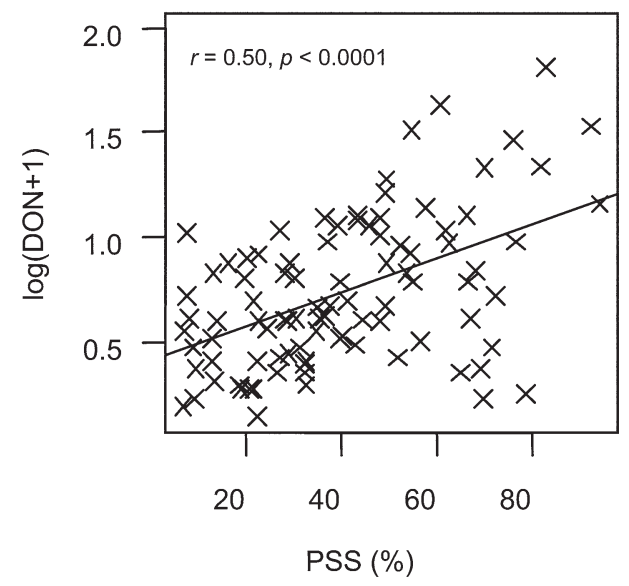

b.

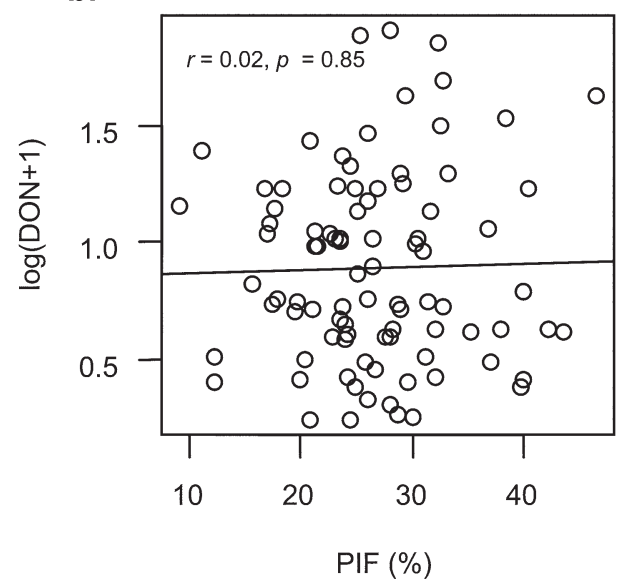

Figure 1. Association between visual disease ratings and deoxynivalenol (DON) contents of the 94 wheat accessions for a) the percentage of symptomatic spikelets (PSS) vs. DON contents from single point-inoculation experiments and $\mathrm{b}$ ) the percentage of infected florets (PIF) vs. DON contents from spraying-inoculation experiments. The lines in $a$ and $b$ are the regression lines.

\section{Data Analysis}

PIF, PSS, and DON content were statistically analyzed using entry means. Entry means of PIF and PSS followed a normal distribution and were used directly for statistical analysis. However, entry means of DON contents deviated from normality and were normalized by logarithmic transformation for further statistical analysis. Correlation coefficients were calculated to estimate the relationships among PIF, PSS, and DON content. Data analysis was conducted using the SAS system for windows v8 (SAS Institute, Inc., Cary, NC). SAS procedure GLM and Duncan's multiple range test were used for analyses of the effects of wheat lines on FHB resistance.

\section{RESULTS}

\section{Variation in Three Types of Resistance}

All inoculated wheat lines showed FHB symptoms after single-point or spray inoculation. Significant differences

in PIF, PSS, and combined DON contents were observed among the accessions with the mean PIF ranging from 9.1 ('Nanda 2419') to $46.5 \%$ (Chanjibaidongmai), the mean PSS ranging from 6.6 (Ning 7840 ) to $92.2 \%$ (Sanyuehuang), and the mean combined DON contents ranging from 0.7 (Ning 7840) to $77.9 \mathrm{mg} \mathrm{kg}^{-1}$ (Chanjibaidongmai) (Table 1). The correlation between PIF and DON data was not significant in 2004 but was very low in $2005(r=0.23, P<0.05)$. Poor correlation of the PIF data was observed between the two spray inoculation experiments. The resistant (Sumai 3) and susceptible controls (Wheaton) showed the same PIF for type I resistance. However, correlation between PSS from the two single-point inoculation experiments was high $(r=0.61, P<0.0001)$, and significant correlations were also observed between the PSS and DON data from the two single-point inoculation experiments. For DON content, significant correlation was observed between the two single-point inoculation experiments $(P<0.05)$, but not between the two spray inoculation experiments (Table 2).

On the basis of the observed levels of type II resistance of previously characterized wheat lines such as Fu 5114, Nobeokabozu, 'Yangmai 4,' and Wheaton (Bai et al., 2003; Liu and Anderson, 2003), the type II resistance levels of the tested wheat accessions were used to assign them into one of four resistance categories: resistant $(\mathrm{R})$, moderately resistant (MR), moderately susceptible (MS), and susceptible (S), which accounted for $27.7,33,28.7$, and $10.6 \%$ of the total

Table 2. Correlation coefficients among FHB infection ratings and deoxynivalenol content (DON) in $\mathrm{mg} \mathrm{kg}^{-1}$ for 94 wheat accessions from different origins based on data from the greenhouse experiments.

\begin{tabular}{|c|c|c|c|c|c|c|c|c|}
\hline Trait & $\begin{array}{c}2003 \text { spring } \\
\text { PSS }^{\dagger}\end{array}$ & $\begin{array}{c}2003 \text { spring } \\
\text { DON }\end{array}$ & $\begin{array}{l}2003 \text { fall } \\
\text { PSS }\end{array}$ & $\begin{array}{l}2003 \text { fall } \\
\text { DON }\end{array}$ & $\begin{array}{c}2004 \\
\text { PIF }\end{array}$ & $\begin{array}{l}2004 \\
\text { DON }\end{array}$ & $\begin{array}{l}2005 \\
\text { PIF }\end{array}$ & $\begin{array}{l}2005 \\
\text { DON }\end{array}$ \\
\hline & $\%$ & $\mathrm{mg} \mathrm{kg}^{-1}$ & $\%$ & $\mathrm{mg} \mathrm{kg}^{-1}$ & $\%$ & $\mathrm{mg} \mathrm{kg}^{-1}$ & $\%$ & $\mathrm{mg} \mathrm{kg}^{-1}$ \\
\hline 2003 spring PSS & - & & & & & & & \\
\hline 2003 spring DON & $0.71^{\star \star \star}$ & - & & & & & & \\
\hline 2003 fall PSS & $0.61^{\star \star \star}$ & $0.48^{\star \star \star}$ & - & & & & & \\
\hline 2003 fall DON & $0.35^{\star \star}$ & $0.28^{*}$ & $0.27^{\star}$ & - & & & & \\
\hline 2004 PIF & 0.07 & 0.19 & 0.09 & 0.13 & - & & & \\
\hline 2004 DON & $0.53^{\star * \star}$ & $0.51^{\star \star \star}$ & $0.65^{\star * *}$ & 0.16 & -0.05 & - & & \\
\hline $2005 \mathrm{PIF}$ & 0.09 & 0.21 & 0.05 & -0.04 & 0.14 & -0.02 & - & \\
\hline 2005 DON & $0.35^{\star \star}$ & $0.29^{\star}$ & $0.32^{* *}$ & $0.36^{\star \star}$ & 0.02 & 0.2 & $0.23^{*}$ & - \\
\hline
\end{tabular}

*Significant at the 0.05 probability level.

${ }^{* \star}$ Significant at the 0.01 probability level.

${ }^{* \star \star}$ Significant at the 0.001 probability level.

${ }^{\dagger} \mathrm{PSS}=$ percentage of symptomatic spikelets; PIF = percentage of infected florets. 
Table 3. Number of accessions and means of percentage of infected florets (PIF), percentage of symptomatic spikelets (PSS), and deoxynivalenol content (DON) content in $\mathrm{mg} \mathrm{kg}^{-1}$ for the 94 wheat accessions in four different FHB resistance categories.

\begin{tabular}{|c|c|c|c|c|c|c|}
\hline \multirow{2}{*}{ Resistance $^{\dagger}$} & \multirow{2}{*}{ Number of accessions } & \multicolumn{2}{|c|}{ Spraying-inoculation } & \multicolumn{2}{|c|}{ Single point-inoculation } & \multirow{2}{*}{ Combined ${ }^{\ddagger}$ DON } \\
\hline & & PIF & DON & PSS & DON & \\
\hline & & $\%$ & $\mathrm{mg} \mathrm{kg}^{-1}$ & $\%$ & $\mathrm{mg} \mathrm{kg}^{-1}$ & $\mathrm{mg} \mathrm{kg}^{-1}$ \\
\hline $\mathrm{R}$ & 26 & $24.2 \mathrm{a}^{\S}$ & $5.1 \mathrm{a}$ & $15.5 \mathrm{a}$ & $3.1 \mathrm{a}$ & $4.0 \mathrm{a}$ \\
\hline MR & 31 & $27.3 \mathrm{a}$ & $6.7 \mathrm{a}$ & $34.5 \mathrm{~b}$ & $6.1 \mathrm{a}$ & $8.3 \mathrm{ab}$ \\
\hline MS & 27 & $28.4 \mathrm{a}$ & $20.8 b$ & $57.3 \mathrm{c}$ & $8.2 \mathrm{a}$ & $14.5 b$ \\
\hline$S$ & 10 & $27.2 \mathrm{a}$ & $22.7 \mathrm{~b}$ & $77.9 d$ & $28.8 \mathrm{~b}$ & $24.9 c$ \\
\hline
\end{tabular}

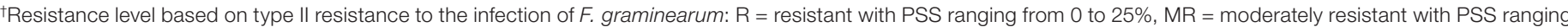
from 26 to 45\%, MS = moderately susceptible with PSS ranging from 46 to $70 \%$, and S = susceptible with PSS ranging from 71 to $100 \%$.

${ }^{\ddagger}$ Mean values based on DON data from both spraying-inoculation and single point-inoculation experiments.

$\S$ Different letter indicates significant difference at $p<0.05$ in Duncan's multiple range test.

wheat accessions, respectively (Table 3). Most of the accessions $(60.7 \%)$ were classified as MR or MS. The mean PSS for the four type II resistance-based categories were significantly different (Table 3). Similar to PSS, the mean DON content of the type II resistance-based $\mathrm{R}$ group was significantly different from the type II resistance-based S group (Table 3). However, the differences were not significant for mean PIFs (type I resistance) among the four categories (Table 3). Four accessions with the highest PIF (PIF > 40\%) demonstrated type II resistance ranging from moderately resistant to susceptible. Under the same conditions, both susceptible (Wheaton) and resistant (Sumai 3) controls had the same PIF, but had significantly different PSS and DON content. The results indicate that type I resistance may not be a stable component of FHB resistance under favorable infection conditions.

The selected resistant or moderately resistant accessions (64 accessions) were all landraces or cultivars from China or Japan except for 'Ernie' and 'Freedom' from the U.S. Seventeen of the twenty-six resistant accessions, including Ning 7840, F 60096, and Minamikyushu 69, showed lower mean DON content than the Sumai 3 control; and nine of the seventeen genotypes had less than $2 \mathrm{mg} \mathrm{kg}^{-1} \mathrm{DON}$ in harvested grain. Among all the accessions examined, four accessions (Huoshaobairimai, Huangfangzhu, Huoshaomai, and Huangcandou) from China and two from Japan (Asozaira III and Nobeokabozu) showed overall resistance with PIF $<28 \%$, PSS $<25 \%$, and DON content $<2 \mathrm{mg}$ $\mathrm{kg}^{-1}$. The mean values of the three resistance traits for the six accessions were superior to those of the resistant control Sumai 3 (Table 1). Five of the six highly resistant wheat landraces, except Nobeokabozu, have not been well studied, and are potentially new sources of FHB resistance.

\section{Association between DON Content and FHB Severity}

Wheat genotypes with low PSS often had relatively low DON content (Tables 1 and 3). A moderately positive correlation was observed between PSS and DON accumulation $(r=0.50, P<0.0001)$ (Fig. 1a). With regard to combined DON content, of the twenty-six accessions that had PSS values less than $25 \%$, nine, including the wellknown FHB-resistant lines Ning 7840, had DON contents of less than $2 \mathrm{mg} \mathrm{kg}^{-1}$, ten, including the well-known Chinese resistant landrace Wangshuibai, had DON contents between 2 and $5 \mathrm{mg} \mathrm{kg}^{-1}$, and the remaining seven had DON contents between 5 and $9.5 \mathrm{mg} \mathrm{kg}^{-1}$. For those moderately resistant accessions, a wide range of DON contents were observed. DON contents of fifteen accessions (48.4\%) were lower than $5 \mathrm{mg} \mathrm{kg}^{-1}$, ten accessions $(32.3 \%)$ were from 5 to $10 \mathrm{mg} \mathrm{kg}^{-1}$, and the remaining six lines (19.3\%) from China showed high DON content. Among the twenty-six moderately susceptible accessions, no accession had DON content lower than $2 \mathrm{mg} \mathrm{kg}^{-1}$, eleven accessions had DON contents between 2 and 10 $\mathrm{mg} \mathrm{kg}{ }^{-1}$, and six accessions were above $20 \mathrm{mg} \mathrm{kg}^{-1}$. The average DON content for susceptible wheat accessions was $25.4 \mathrm{mg} \mathrm{kg}^{-1}$ with only one accession (Kuangtuerhhsiaomai) that had a DON content of less than $2 \mathrm{mg} \mathrm{kg}^{-1}$ (Table 3). The accession with the highest DON content (Chanjibaidongmai) was in the susceptible group based on observed levels of type II resistance (Table 1).

There was no correlation between PIF and DON content in spray inoculation experiments $(r=0.02, P=$ $0.85)$ (Fig. 1b). Fifty-two accessions had lower PIF (<28\%) than both the resistant (Sumai 3) and the susceptible controls (Wheaton). Interestingly, among the four accessions with the highest PIF $(>40 \%)$, three accessions had about $3 \mathrm{mg} \mathrm{kg}^{-1}$ of DON and only Youzimai had a high DON content of $46.6 \mathrm{mg} \mathrm{kg}^{-1}$.

\section{DISCUSSION}

All resistant and moderately resistant accessions were collected from China except for Chokwang (Korea) and Ernie and Freedom (U.S.). Nine of twenty-six accessions showing resistant type II reactions had DON contents lower than $2 \mathrm{mg} \mathrm{kg}^{-1}$. Fu 5114 and Ning 7840 share the same source of resistance (Yu et al., 2006). Five other wheat accessions that showed a high level of type II resistance and low DON have not been well studied. Four of the five accessions are 
landraces from China, and one is a landrace from Japan. Available literature could not reveal any known genetic relationship between them and Sumai 3 (Yu et al., 2006), suggesting that these wheat lines may have novel QTL for FHB resistance and low DON content. Further mapping work may facilitate identification of potential new QTL for FHB resistance in these landraces.

The relationship between FHB severity and DON content is still unknown. Some studies demonstrated a high positive correlation between FHB severity and DON content (Lemmens et al., 1994; Wang and Miller, 1988), while others reported poor or no correlation between the two traits (Mesterházy et al., 1999; Wiśniewska et al., 2004). These contradictory reports might have resulted from inoculating plants at different stages of plant growth, from using different methods for inoculation, from differences in methods of harvesting infected grain and DON analysis, and from sampling accessions with different ranges of FHB resistance, etc. For example, if FHB infection starts at heading or early anthesis, kernels in an infected spike may stop development at an early stage. The heavily infected kernels are too small to be collected. A susceptible cultivar may show much lower DON content than it should have because severely infected kernels are not included in DON analysis. On the other hand, if infection occurs after anthesis, normal sized infected kernels are collected in threshed grains for DON analysis. For the same reason, single-point inoculation may underestimate the DON content compared to spray inoculation or grain spawn inoculation on the soil surface in field conditions because single-point inoculation may facilitate early infection that may prevent kernel development, especially when a threshing machine is used. Careful hand threshing may keep more kernels with high DON content while a threshing machine may blow away severely infected kernels that contain most of the toxin.

Results of this study showed that type II resistance was moderately correlated with DON content. However, no correlation was observed between type I resistance and DON content, which agrees with the result of Lemmens et al. (2005) and others (Mesterházy et al., 1999; Wiśniewska et al., 2004). In general, the resistant group had the lowest mean DON content and the susceptible group had the highest. However, large variation in DON content was still observed within each group, especially for the moderately susceptible group (ranging from 1 to $46.6 \mathrm{mg} \mathrm{kg}^{-1}$ ).

Mild visual FHB symptoms on the infected spikes (type II resistance) usually indicates low DON contamination, but visual FHB symptoms may not always correlate with the levels of DON content for wheat lines with moderate FHB type II resistance. Fourteen lines in this study had DON content less than $2 \mathrm{mg} \mathrm{kg}^{-1}$, and five of them were moderately resistant. Wheat landrace Kuangtuerhhsiaomai was susceptible with $71.4 \%$ PSS, but showed low DON content of $1.6 \mathrm{mg} \mathrm{kg}^{-1}$. The low DON content in Kuangtuerhhsiaomai might be a result of type III resistance or result from poor seed-setting due to heavy infection. Many infected kernels were not collectable for DON analysis. In contrast, landrace Meiqianwu showed a low PSS (16.1\%) but relatively high $\left(9.5 \mathrm{mg} \mathrm{kg}^{-1}\right)$ DON content.

It appears that three types of FHB resistance are present in these wheat lines and assessment of individual resistance components in these sources will facilitate their use in breeding FHB-resistant wheat cultivars. Resistance to FHB is most effective when a wheat line carries all three types of resistance (Kolb et al., 2001). However, the effects of the three types of resistance on cultivar overall FHB resistance may not be the same. Type I resistance may involve escape from FHB infection by a passive mechanism, such as a narrow flower opening (Gilsinger et al., 2005; Mesterházy, 1995). To date, type I resistance has not been well characterized in wheat and it may not be a reliable component to select for FHB resistance in breeding programs. DON has been proposed as a virulence factor that plays an important role in the spread of FHB in a wheat spike (Bai et al., 2001a), and wheat resistance to DON accumulation also has been found closely correlated with type II resistance in this study and other previous studies (Bai et al., 2001b; Lemmens et al., 2005). In general, a cultivar with high type II resistance most likely has a low DON content. Selection for a genotype with a high level of type II resistance may indirectly select for low DON. Hence, selection for type II resistance should be the first priority in breeding for overall FHB resistance. For moderately resistant or moderately susceptible cultivars, it may be more important to select for both type II resistance and lower DON genotypes to reduce DON content in new cultivars.

\section{References}

Bai, G.-H., and G.E. Shaner. 1994. FHB of wheat: Perspective and control. Plant Dis. 78:760-766.

Bai, G.-H., and G.E. Shaner. 1996. Variation in Fusarium graminearum and cultivar resistance to wheat scab. Plant Dis. 80:975-979.

Bai, G.-H., A.E. Desjardins, and R.D. Plattner. 2001a. Deoxynivalenol-nonproducing Fusarium graminearum causes initial infection, but does not cause disease spread in wheat spikes. Mycopathologia 153:91-98.

Bai, G.-H., R.D. Plattner, A.E. Desjardins, and F.L. Kolb. 2001 b. Resistance to Fusarium head blight and deoxynivalenol accumulation in wheat. Plant Breed. 120:1-6.

Bai, G.-H., P.-G. Guo, and F.L. Kolb. 2003. Genetic relationships among head blight resistant cultivars of wheat assessed on the basis of molecular markers. Crop Sci. 43:498-507.

Bai, G.-H., and G.E. Shaner. 2004. Management and resistance in wheat and barley to Fusarium head blight. Annu. Rev. Phytopathol. 42:135-161.

Desjardins, A.E., R.H. Proctor, G.H. Bai, S.P. McCormick, G. Shaner, G. Buechley, and T.M. Hohn. 1996. Reduced virulence of trichothecene antibiotic-nonproducing mutants of 
Gibberella zeae in wheat field tests. Mol. Plant Microbe Interact. 9:775-781.

Dexter, J.E., and T.W. Nowicki. 2003. Safety assurance and quality assurance issues associate with Fusarium head blight in wheat. p. 420-60. In K.J. Leonard and W.R. Bushnell (ed.) Fusarium head blight of wheat and barley. APS Press, St. Paul, MN.

Gilsinger, J., L. Kong, X. Shen, and H. Ohm. 2005. DNA markers associated with low Fusarium head blight incidence and narrow flower opening in wheat. Theor. Appl. Genet. 110:1218-1225.

Kolb, F.L., G.-H. Bai, G.J. Muehlbauer, J.A. Anderson, K.P. Smith, and G. Fedak. 2001. Host plant resistance genes for Fusarium head blight: Mapping and manipulation with molecular markers. Crop Sci. 41:611-619.

Lemmens, M., A. Reisinger, H. Buerstmayr, and P. Ruckenbauer. 1994. Breeding for head blight (Fusarium spp.) resistance in wheat: Development of a mycotoxin-based selection method of seedlings. Acta Hortic. 355:223-232.

Lemmens, M., U. Scholz, F. Berthiller, C. Dall'Asta, A. Koutnik, R. Schuhmacher, G. Adam, H. Buerstmayr, A. Mesterházy, R. Krska, and P. Ruckenbauer. 2005. The ability to detoxify the mycotoxin deoxynivalenol colocalizes with a major quantitative trait locus for Fusarium head blight resistance in wheat. Mol. Plant Microbe Interact. 18:1318-1324.

Liu, S., and J.A. Anderson. 2003. Marker-assisted evaluation of Fusarium head blight-resistant wheat germplasm. Crop Sci. 43:760-766.

Luo, Y., T. Yoshizawa, and T. Katayama. 1990. Comparative study on the natural occurrence of Fusarium mycotoxins (trichothecenes and zearalenone) in corn and wheat from high- and low-risk areas for human esophageal cancer in China. Appl. Environ. Microbiol. 56:3723-3726.

Marasas, W.F.O., P.E. Nelson, and T.A. Toussoun. 1984. Toxigenic Fusarium species: Identification and Mycotoxicology. Pennsylvania State Univ. Press, University Park, PA.

McMullen, M., R. Jones, and D. Gallenberg. 1997. Scab of wheat and barley: A re-emerging disease of devastating impact. Plant Dis. 81:1340-1348.

Mesterházy, Á. 1995. Types and components of resistance to Fusarium head blight of wheat. Plant Breed. 114:377-386.

Mesterházy, Á., T. Bartók, C.G. Mirocha, and R. Komoróczky.
1999. Nature of resistance to Fusarium head blight and the role of deoxynivalenol for breeding. Plant Breed. 118:97-110.

Miedaner, T., B. Schneider, and H.H. Geiger. 2003. Deoxynivalenol (DON) content and Fusarium head blight resistance in segregating populations of winter rye and winter wheat. Crop Sci. 43:519-526.

Miller, J.D., J.C. Young, and R.D. Sampson. 1985. Deoxynivalenol and Fusarium head blight resistance in spring cereals. Phytopathol. Z. 113:359-367.

Mirocha, C.J., E. Kolaczkowski, W. Xie, H. Yu, and H. Jelen. 1998. Analysis of deoxynivalenol and its derivatives (batch and single kernel) using gas chromatography/mass spectrometry. J. Agric. Food Chem. 46:1414-1418.

Parry, D.W., P. Jenkinson, and L. McLeod. 1995. Fusarium ear blight (scab) in small grain cereals, a review. Plant Pathol. 44:207-238.

Rudd, J.C., R.D. Horsley, A.L. McKendry, and E.M. Elias. 2001. Host plant resistance genes for Fusarium head blight: Sources, mechanisms, and utility in conventional breeding systems. Crop Sci. 41:620-627.

Schroeder, H.W., and J.J. Christensen. 1963. Factors affecting resistance of wheat to scab caused by Gibberella zeae. Phytopathology 53:831-838.

Shaner, G.E., and G. Buechley. 2003. Relation between head blight and grain quality in the Indiana FHB epidemic of 2003. p. 178-181. In S.M. Canty et al. (ed.) Proc. of the Natl. Fusarium Head Blight Forum, Bloomington, MN. 13-15 Dec. 2003. Mich. State Univ., East Lansing, MI.

Snijders, C.H.A. 1990. Fusarium head blight and mycotoxins contamination of wheat, a review. Neth. J. Plant Pathol. 96:187-198.

Wang, Y.-Z., and J.D. Miller. 1988. Effects of Fusarium graminearum metabolites on wheat tissue in relation to FHB resistance. J. Phytopathol. 122:118-125.

Wiśniewska, H., J. Perkowski, and Z. Kaczmarek. 2004. Scab response and deoxynivalenol accumulation in spring wheat kernels of different geographical origins following inoculation with Fusarium culmorum. J. Phytopathol. 152:613-621.

Yu, J.-B., G.-H. Bai, S.-B. Cai, and T. Ban. 2006. Marker-assisted characterization of Asian wheat lines for resistance to Fusarium head blight. Theor. Appl. Genet. 113:308-320. 